only 10 per cent every 20 days. As a result, virtually all oocytes have disappeared from the ovaries of the $C 3 H$ or $C B A$ strains by the age of 450 days, when there are still about 500 in the ovaries of an $A$ strain mouse. Transplantation from one strain to another should make it possible to determine the extent to which these differences depend on inherent differences in the ovaries, and how much on the environment, particularly the endocrine system and its secretions.

Second, it extends the scope of many experiments which hitherto have depended on and been limited by the technique of tubal transfer of ova. The successful transfer of an ovary of one genotype into the environment of another genotype should theoretically provide opportunities for the transformation of the genetic and cytoplasmic structure either of oocytes while they lie dormant in the early prophase stage of meiotic division or, if embryonic ovaries are used, of oogonia which are still dividing mitotically. $C 3 H$ or $C B A$ offspring born in this new way to $A$ strain mothers will not only have been exposed to an $A$ strain environment throughout intra-uterine life but also for any desired. length of time while the primordial or developing oocytes remain within the grafted ovary.

This work has been carried out during the tenure of a Nuffield Senior Gerontological Research Fellowship.

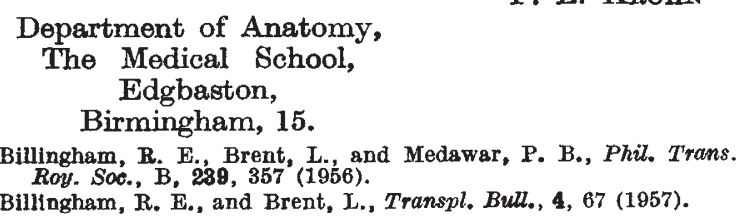

\section{An Unstable Mutation in Salmonella typhimurium}

RECENTLY, Zamenhof et al. ${ }^{1}$ described experiments which demonstrated the occurrence of pinpoint mutant colonies in Escherichia coli. These mutants arose in experiments in which 5-bromo-uracil was used as a mutagenic agent, and the pinpoint colonies were shown to be of three types: stable, unstable and very unstable. The unstable pinpoints were maintained for seven transfers, while the very unstable pinpoints were lost after only $12 \mathrm{hr}$. of growth.

I have recently demonstrated that similar unstable mutations occur spontaneously in Salmonella typhimurium. The mutations studied were from 'leucineless' to 'leucine independent'. It thus appears that the occurrence of unstable mutations in bacteria is not limited to $E$. coli, or to experiments in which a mutagenic agent is used.

In $S$. typhimurium mutations of 'leucineless' to 'leucine independent' are of three types distinguishable by their rate of growth on medium lacking leucine. The three types have been called 'fast', 'slow' and 'very slow'. The fast type grows to full colony size in $24 \mathrm{hr}$. (as does the wild type). The slow type shows barely visible growth after $24 \mathrm{hr}$. but has grown to full colony size after $48 \mathrm{hr}$. The very slow type shows barely visible growth after 48 $\mathrm{hr}$. but has grown to a full-size colony after $72 \mathrm{hr}$. On medium containing leucine all three types reach full colony size after $24 \mathrm{hr}$. incubation.

The fast and slow types are both stable and can be transduced into the leucineless strain yielding only fast and slow transductional clones respectively.
The very slow type is unstable in that it reverts spontaneously to leucineless at a very high rate. This instability is detected by shaking the clone to be tested in saline, diluting suitably, and spreading equal samples, each containing about 500 cells, on plates with and without leucine, and incubating the plates for $72 \mathrm{hr}$. The instability is then demonstrated first by there being fewer colonies on the plates without leucine than on those with leucine, and secondly those colonies which do develop on the plates without leucine show a gradation of sizes from minute to $4 \mathrm{~mm}$. in diameter. All the colonies on the plates with leucine are of equal size.

Very slow clones arising from independent mutations show varying degrees of instability. One clone was shaken in saline and plated on medium lacking leucine. All the colonies that grew were very slow. Eight of these colonies were shaken in saline and samples plated on medium with leucine and on medium without leucine. It was found that all eight colonies contained 1-20 per cent of very slow cells (80-99 per cent leucineless cells). From a similar experiment on a different clone twenty colonies were shown to contain 0-0.2 per cent of very slow cells (99.8100 per cent leucineless cells).

The fast type is probably a true back-mutation of leucineless to wild type; the slow type may be due to a suppressor mutation or to a mutation to a different allele at the leucine locus.

Further experiments are in progress to determine whether the instability is confined to the leucine locus, and whether a leucineless strain obtained from the very slow type shows any residual instability.

School of Botany,

Trinity College,

University of Dublin. April 20.

${ }^{2}$ Zamenhof, S., de Giovanni, R., and Greer, S., Nature, 181, 827 (1958).

\section{Effects of Gibberellic Acid on Nicotiana Plants}

THE physiological action and practical application of gibberellic acid are well known (H. Seltman, private communication and refs. 1 and 2). Tests were conducted to investigate its possible application to the control of the alkaloid content and plant development of different Nicotiana species. Gibberellic acid was supplied by Eli Lilly and Co. and Merck and Co.

Seedling survival and new growth. Gibberellic acid was tested to see whether it can help tobacco seedlings to overcome the shock immediately following transplanting to the field and thus improve survival of seedlings and stimulate earlier growth. Immersion of the root in 10 p.p.m. gibberellic acid for $10 \mathrm{~min}$. before transplanting, or spraying with I p.p.m. gibberellic acid on the leaves after transplanting to the field had no observable effect.

Shoot development. N. tabacum var. Robinson and var. Maryland Mammoth, N. tomentosa, N. tomentosiformis, $N$. otophora and $N$. wigandioides were employed to study the effects of gibberellic acid on the development of leaf, stem and flower. Five of these plants were each given six applications of $15 \mu \mathrm{gm}$. gibberellic acid by injection with a hypodermic needle near the growing point at weekly intervals beginning two weeks after transplanting. At 10 weeks the treated plants were approximately one-third taller than the five controls, due to an 